\title{
Phase Evolution of Cathode Materials in Contact with Ceramic Electrolytes in Solid-State Batteries
}

\author{
Jung-Hyun Kim, ${ }^{\text {a }}$ Chan-Yeop Yu, ${ }^{a}$ Jun-Bin Choi, ${ }^{a}$ and Venkataramani Anandan ${ }^{b}$ \\ aDepartment of Mechanical and Aerospace Engineering, The Ohio State University, \\ Columbus, Ohio, USA (kim.6776@osu.edu) \\ bEnergy Storage Research Department, Research and Innovation Center, Ford Motor \\ Company, Dearborn, Michigan, USA
}

\begin{abstract}
Recently, significant research efforts have been devoted on the development of Liconducting ceramic electrolytes and all solid-state Li-ion battery system, with a goal to improve Li-ion battery safety by eliminating flammable liquid electrolytes. Among various solid electrolytes investigated, $\mathrm{Li}_{6.7} \mathrm{La}_{3} \mathrm{Zr}_{1.7} \mathrm{Ta}_{0.3} \mathrm{O}_{12}$ (LLZT) with garnet structure and

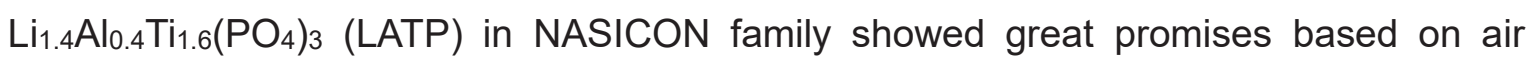
stability, good mechanical properties, and reasonable Li-ion conductivity $\left(10^{-4}-10^{-5}\right.$ $\mathrm{S} / \mathrm{cm})$.
\end{abstract}

The main barrier of achieving stable performances from all solid-state Li-ion battery, however, is the instability of electrode - electrolyte interface. In particular, fabrication process of solid-state batteries involves high-temperature adhesion between ceramic components in the cells. During the sintering process, elemental inter-diffusion becomes facilitated, and this leads to a formation of secondary phases at interfaces (i.e., cathode - electrolyte). These secondary phases often have transport properties and electrochemical performances inferior to the parent materials and thereby degrade the cell performance significantly.

In this presentation, we will report a systematic study on the phase evolution of various cathode materials (e.g., $\mathrm{LiCoO}_{2}$, $\mathrm{LiNiO}_{2}, \mathrm{LiNi}_{1 / 3} \mathrm{Co}_{1 / 3} \mathrm{Mn}_{1 / 3} \mathrm{O}_{2}, \mathrm{LiNi}_{0.5} \mathrm{Mn}_{1.5} \mathrm{O}_{4}$, and $\mathrm{LiFePO}_{4}$ ) in contact with selected solid-state electrolytes (e.g., LLZT or LATP) at high temperatures $\left(500-900^{\circ} \mathrm{C}\right)$. By XRD and Rietveld refinement analysis, we explored the (1) on-set temperatures of unwanted side-reactions between solid-electrolytes and cathodes, (2) types of secondary phases and their evolutions depending on temperature, and (3) fundamental reaction mechanisms between different types of solid electrolytes and cathode materials. This structural information was compared with electrochemical properties to find the phase stability - microstructure - electrochemical performances relationship, which would serve as a prerequisite for designing new solid electrolyte cathode interfaces in future studies. 